\title{
Performance Observation of Distinct Routing Protocols under IPV6 Environment
}

\author{
Shival Chadda \\ Dept. of Computer Science \\ Lord Krishna Polytechnic \\ College, Kapurthala
}

\begin{abstract}
In a mobile ad hoc network (MANET), the topology of the network may change rapidly and unexpectedly due to mobility of nodes. Routing protocols operate differently under distinct environments. Thus, it is necessary to analyze the behavior of different routing protocols under distinct environments. Thus, setting up routes that meet high reliability is a very challenging issue. Another important problem in the MANETs is the energy consumption of nodes. Our study is more concentrated on IPv6 than IPv4. The IPv6-enabled network architecture will become the future standard. IPv6 based network is more protected than IPv4 network and also IPv6 has huge address space support. In this Paper study has been done on the performance observation of various routing protocols of MANET under IPv6 Environment .OPNET Modeler 14.5 is used as simulation tool. We have analyzed the behavior of three routing protocols in the network protocol IPV4 \& IPV6 and compared the performance of these protocols using OPNET Modeler 14.5.IPv6 Migration planner helps in migrating the nodes to IPv6 Environment. Routing protocols are estimated under IPv6 environment on the basis of Wireless LAN Load, wireless LAN Data dropped, Throughput, and Network load. The objective of this research is to examine how these routing protocols act under IPv6 environment and identify which routing protocol performs better.
\end{abstract}

\section{General Terms}

\section{MANET}

\section{Keywords}

Mobile Adhoc Networks, Routing Protocols, AODV, OLSR, GRP, Internet Protocol version 6.

\section{INTRODUCTION}

A mobile ad hoc network lacks a fixed infrastructure and has a dynamically changing topology. The nodes move freely and independently of one another. Ad hoc networks are heavily used in emergency situations where no infrastructure is available, for e.g. battle fields, disaster mitigation etc. The main limitation of ad-hoc systems is the Availability of power. In addition to running the onboard electronics, power consumption is governed by the number of processes and overheads required to maintain connectivity [1]. MANET is a network consists of many nodes which communicate to each other without any central control authority. Bandwidth, energy, physical security and other resources are limited in mobile ad hoc network. In mobile ad hoc network every mobile node acts as a host and also as a router.

\section{INTERNET PROTOCOL VERSION 6}

With the rapid development in wireless communications in recent years, the necessity for sufficient Internet protocol (IP) addresses to meet the demand of mobile devices, as well as flexible communications without infrastructure, are especially considerable. The next-generation IP, Internet Protocol version 6 (IPv6) [1], [2], provides sufficient IP addresses to enable all kinds of devices to connect to the Internet and promotes mobile wireless commerce (m-commerce). The IPv6-enabled network architecture will become the future standard.

Additionally, most current mobile devices are equipped with IEEE 802.11 wireless local area network (WLAN) interface cards. IEEE 802.11 WLAN supports two operating modes: infrastructure mode and ad hoc mode. The infrastructure mode requires all mobile devices to directly communicate to the access point (single-hop communication). In the ad hoc mode, mobile devices dynamically form a mobile ad hoc network (MANET) with multi-hop routing. Clearly, the ad hoc mode allows for a more flexible network, but its aim is not to connect to the Internet. In this paper, we address the issue of connecting MANETs to global IPv6 networks while supporting IPv6 mobility with various routing protocols.

Much attention has been paid to IP address auto configuration and IPv6 extension for MANETs [3]-[5] in recent years. IPv6 auto configuration mechanism [3], [4] allows a node to generate a link-local IP address. Extension has also been made to be suitable for MANET [5]. However, global connectivity for a mobile node is not supported in [5]. Later on, [6] and [7] address how to provide global connectivity for an IPv6enabled MANET. In these works, a MANET node can acquire a global IPv6 address from an Internet gateway, and then access to the Internet through the gateway. Routing in MANETs and the IPv6 network is based on existing protocol.

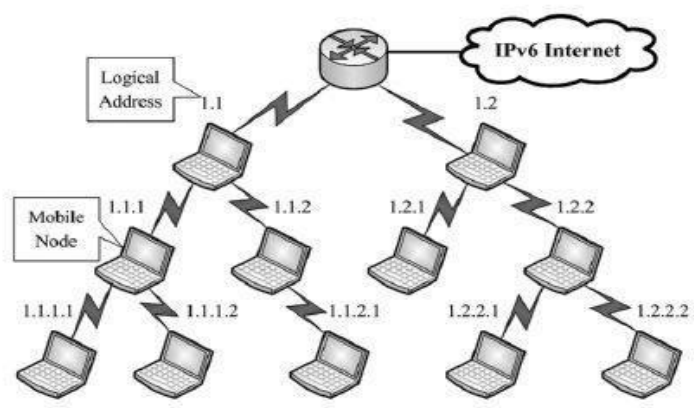

Figure 1: A self-organizing, self-addressing, self-routing IPv6-based MANET. [14]

\section{ROUTING PROTOCOLS IN MANET}

The routing protocols that are available for MANET comprise 
- Proactive (table driven).

- Reactive (on demand).

- Hybrid routing protocols.

Popular proactive routing protocols are highly dynamic Destination- Sequenced Distance Vector (DSDV) and Optimized Link State Routing protocol (OLSR) while reactive routing protocols include Ad hoc on demand Distance Vector (AODV) and Dynamic Source Routing (DSR). An example of a hybrid routing protocol is Zone Routing Protocol (ZRP).

\subsection{AODV}

AODV is an on demand routing protocol, that is, it builds routes between nodes only as desired by source nodes. It maintains these routes as long as they are needed by the sources [9]. Nodes maintain a route cache and use a destination sequence number for each route entry. The fact that a node in AODV seeks information about the network only when needed reduces overhead since nodes do not have to maintain unnecessary route information while the use of a sequence number ensures loop freedom. This paper [9] discusses the effects of packet size on the AODV routing protocol implementation in homogenous and heterogeneous MANET.

\subsection{OLSR}

OLSR is a proactive or table driven, link-state routing protocol. Link-state routing algorithms choose best route by deciding various characteristics like link load, delay, bandwidth etc. Link-state routes are more reliable, stable and accurate in calculating best route and more problematic than hop count. To update topological information in each node, periodic message is broadcast over the network. Multipoint relays are used to facilitate efficient flooding of control message in the network. Route calculations are done by multipoint relays to form the rout from a given node to any destination in the network. The OLSR protocol is developed to work individually from other protocols .OLSR contain three generic elements: a mechanism for neighbor sensing, a mechanism for efficient flooding of control traffic, and a specification of how to select and diffuse tolerable topological information in the network in order to prove excellent routes [18].

\subsection{GRP}

GRP PROTOCOL (Gathering based routing protocol) protocol is source initialized protocol in MANET routing protocol in which all the routing path is created by source node in Mobile Ad-hoc network. In this protocol, source node collects all the information about the route to the designation. In this procedure, source node sends a destination Query toward the destination through network. It works like AODV and DSR using RREQS (Reverse Request Query by Source). In it, when destination Query reached to the destination, destinations send a packet called Network Information Gathering (NIG) which approach through network. When NIG packet reached at a router, router gives it all the information about the network and its resources. There are many nodes called Effective Outgoing Links (EIL) where NIG packet does not riches, routers send this information to these EILs. At last NIG reaches at source node and source node get all the information [19]

\section{RELATED WORK}

$\operatorname{In}[20]$, the author study two routing protocols AODV and OLSR on the basis of various parameters like performance and scalability, security, resource usage and has drawn the conclusion about AODV and OLSR, and on the basis of this study and has recommended the appropriate routing protocol which is convenient under different situations. Razan Al-Ani [21] describes the characteristics of Mobile Ad hoc Networks (MANETs), and their Routing protocol, and second a mobile ad hoc network (MANET) which consists of set mobile wireless nodes and one fixed wireless server are design using OPNET Modeler 14.5. The performance of this network under different routing protocol is evaluated by three metrics: delay, network load and throughput. In [22], the author clarify how mobility is affected by the two mobile ad-hoc routing protocols. In this study, he first examine the two routing protocols under static condition linear fashion, and then under mobility. This helps to know how rapid modification in topology may alter routing protocols. In [23] author compares the AODV and OLSR routing protocols in small number of nodes $(10,15$, and 20$)$ under ipv6 environment. In the end result are compared by metrics network load, throughput, and 'end to end delay.

\section{SIMULATION ENVIRONMENT AND PARAMETERS}

The research is carried out using discrete event simulation software known as OPNET (Optimized Network Engineering Tool) Modeler version 14.5. It is one of the most widely used commercial simulators based on Microsoft Windows platform and incorporates more MANET routing parameter as compared to other commercial simulator available. It not only supports MANET routing but also provides a parallel kernel to support the increase in stability and mobility in the network. [16] Claims that OPNET's intensive analyzing feature provides best environment for analyzing and integrating the output obtained.

The simulations focussed on the performance of routing protocols in the ipv6 environment. There are three simulation scenarios consisting of 75 nodes and 150 nodes. The nodes were randomly placed within certain gap from each other in 10000x10000 square meters campus Environment for 75 nodes and 150 nodes.

\subsection{Simulation Environment}

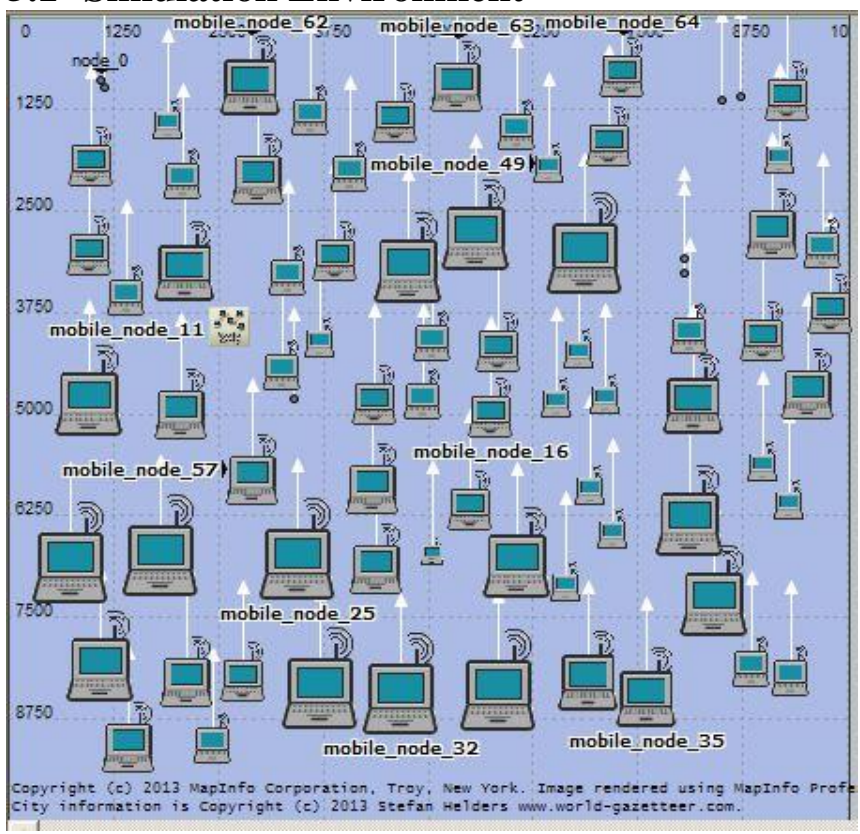

Figure 2.Simulation Scenario Having 75 Nodes 


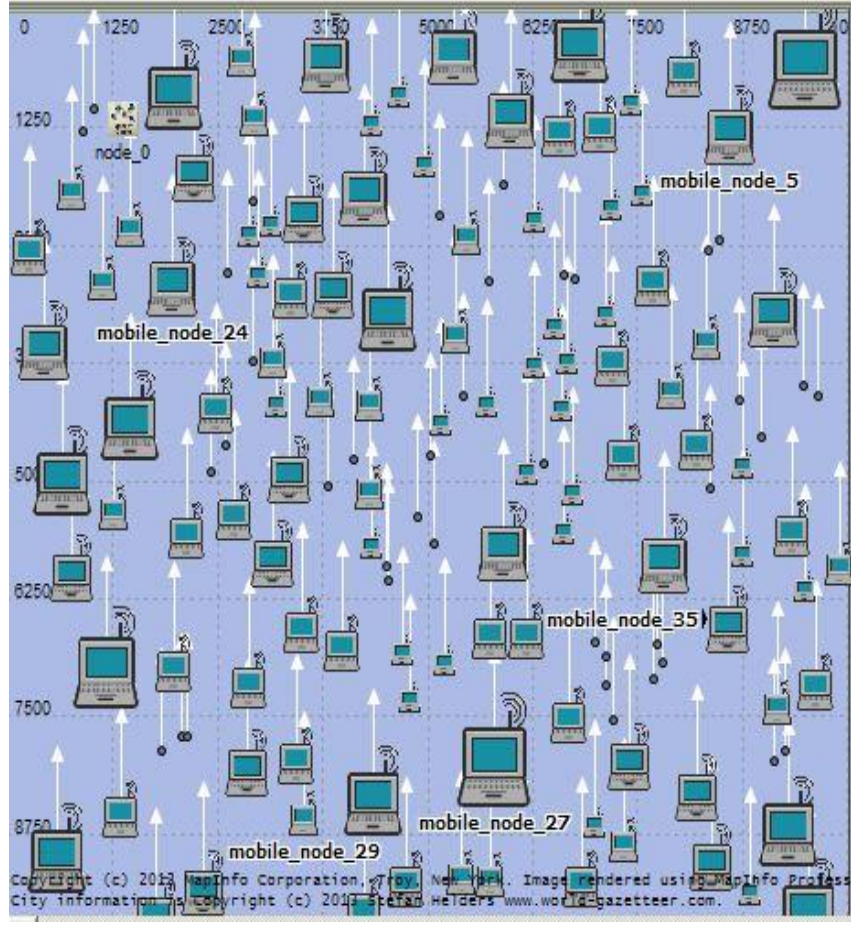

Figure 3.Simulation Scenario Having 150 Nodes

Table 1: Simulation Settings

\begin{tabular}{|c|c|}
\hline Parameter & Value \\
\hline Simulator & OPNET 14.5 \\
\hline Simulation time & $\mathbf{1 0 ~ m i n}$ \\
\hline Number of nodes & $\mathbf{7 5 , 1 5 0}$ \\
\hline Data rate & $\mathbf{1 1}$ Mbps \\
\hline Environment size & $\mathbf{1 0 0 0 0 * 1 0 0 0 0}$ meters \\
\hline Traffic type & $\begin{array}{c}\text { Constant Bit Rate } \\
\text { (CBR) }\end{array}$ \\
\hline Packet size & $\mathbf{5 1 2}$ \\
\hline Network protocol & IPv4 ,IPv6 \\
\hline Transport Protocol & TCP \\
\hline Routing Protocol & AODV,OLSR,GRP \\
\hline Propagation Model & Random Way Point \\
& Model \\
\hline MAC protocol & $\mathbf{8 0 2 . 1 1}$ \\
\hline Simulation kernel & Optimized \\
\hline
\end{tabular}

\subsection{Wireless Parameters}

The Wireless LAN parameters were common to all of routing protocols The wireless LAN parameters configured matches to research work of [17], except the buffer size was set to 204800000 bits as heavier flow of application was generated. In addition, the channel settings were set to "auto assigned" in order to avoid manual error. Also the transmission power was changed from 0.005 watt to 0.050 watt.

Table 2: Wireless LAN Parameters

\begin{tabular}{|c|c|}
\hline Parameter & Value \\
\hline Wireless LAN MAC Address & Auto Assigned \\
\hline
\end{tabular}

\begin{tabular}{|c|c|}
\hline BSS Identifier & Auto Assigned \\
\hline Physical Characteristics & Direct Sequence \\
\hline Data Rate (bps) & 11 Mbps \\
\hline Channel Settings & Auto Assigned \\
\hline Transmit Power & $\mathbf{0 . 0 5 0}$ \\
\hline RTS Threshold & None \\
\hline Packet-Reception Threshold & $\mathbf{- 9 5}$ \\
\hline Short Retry Limit & $\mathbf{7}$ \\
\hline $\begin{array}{c}\text { Long Retry Limit } \\
\text { AP Beacon Interval (seconds) }\end{array}$ & $\mathbf{4 0 2}$ \\
\hline $\begin{array}{c}\text { Max Receive Lifetime } \\
\text { (seconds) }\end{array}$ & $\mathbf{0 . 5}$ \\
\hline Buffer Size (bits) & $\mathbf{2 0 4 8 0 0 0 0}$ \\
\hline $\begin{array}{c}\text { Large Packet Processing } \\
\text { HCF }\end{array}$ & Fragment \\
\hline
\end{tabular}

\subsection{Routing Protocol Parameters}

The configuration parameter for AODV was setup as in the work of [17] except Time-To -Live (TTL) was set to default configuration as set by OPNET Modeler 14.5

\section{Table 3: AODV Parameters}

\begin{tabular}{|c|c|}
\hline Route Request Retry & $\mathbf{5}$ \\
\hline $\begin{array}{c}\text { Route Request Rate } \\
\text { Limits } \\
\text { (pkts/sec) }\end{array}$ & $\mathbf{1 0}$ \\
\hline $\begin{array}{c}\text { Gratuitous Route } \\
\text { Reply Flag }\end{array}$ \\
\hline $\begin{array}{c}\text { Active Route } \\
\text { Timeout (seconds) }\end{array}$ & $\mathbf{3 0}$ \\
\hline $\begin{array}{c}\text { Hello Interval } \\
\text { (seconds) }\end{array}$ & Uniform(10,10.1) \\
\hline $\begin{array}{c}\text { Allowed Hello Loss } \\
\text { Timeout Buffer }\end{array}$ & $\mathbf{1 0}$ \\
\hline Addressing Mode & IPv4, IPv6 \\
\hline
\end{tabular}


Table 4: OLSR Parameters

\begin{tabular}{|c|c|}
\hline Willingness & Always \\
\hline Hello Interval (Seconds) & $\mathbf{2 . 0}$ \\
\hline TC Interval (Seconds) & $\mathbf{5 . 0}$ \\
\hline $\begin{array}{c}\text { Neighbor Hold Time } \\
\text { (Seconds) }\end{array}$ & $\mathbf{6 . 0}$ \\
\hline $\begin{array}{c}\text { Topology Hold Time } \\
\text { (Seconds) }\end{array}$ & $\mathbf{1 5 . 0}$ \\
\hline $\begin{array}{c}\text { Duplicate Message Hold } \\
\text { Time (Seconds) }\end{array}$ & $\mathbf{3 0 . 0}$ \\
\hline Addressing Mode & IPv4, IPv6 \\
\hline
\end{tabular}

Table 5: GRP Parameters

\begin{tabular}{|c|c|}
\hline Hello Interval (seconds) & Uniform(4.9,5.0) \\
\hline Neighbor Expiry Time & Constant (10) \\
\hline Distance Moved(meters) & $\mathbf{1 0 0 0}$ \\
\hline $\begin{array}{c}\text { Position Request } \\
\text { Timer(seconds) }\end{array}$ & $\mathbf{5 . 0}$ \\
\hline Backbone Option & Enabled \\
\hline Routes Export & $\mathbf{1}$ \\
\hline Number of Initial Floods & IPv4, IPv6 \\
\hline Addressing Mode &
\end{tabular}

\subsection{DES Configuration Parameter}

The DES simulation criterion was construct as similar to the manual provided by [1] and was run for total time of 600 seconds. The overall simulation was monitored within the following criteria:

- Duration: 10 minutes ,Seed: 256

- Update Interval: 500000 events. (This specifies how often simulation calculates events/second data.)

- Simulation Kernel: Optimized ('Optimized' kernel was chosen because it high-velocity than the remaining other two simulation kernel.)

\section{Performance Metrics}

In order to evaluate the performances of three MANET protocols, several metrics need to consider. These metrics reflect how efficiently the data is delivered. In epidemic routing, multiple copies may be delivered to the destination. According to the literatures [10], [11], [12] and [13], some of these metrics are suggested by the MANET working group for routing protocol evaluation.

\subsection{Data Packet Dropped}

The routers might fail to deliver or drop some packets or data if they arrive when their buffer are already full. Some, none, or all the packets or data might be dropped, depending on the state of the network, and it is impossible to determine what will happen in advance.

\subsection{Routing Load}

The total number of routing packets transmitted during the simulation. For packets sent over multiple hops, each transmission of the packet or each hop counts as one transmission.

\subsection{Average Throughput (TP)}

Average Throughput [24] is the number of bytes received successfully and is calculated by

Average Throughput $=($ Number of bytes received $x$ 8) $/$ (Simulation time $x$ 1000) Kbps

\subsection{Network Load}

Network load refers to the amount of data being carried by the network.Network load shows the bit/sec load submitted by all higher layers in all WLAN nodes of the network to wireless LAN layers. The adequate network should come up with this heady traffic load and provide finest network infrastructure and many techniques are used for this. Heavy load on network may affect mobile ad hoc networks the data packets may collide. This increases congestion on the network and makes the routing process slow.

\section{SIMULATION RESULTS AND} OBSERVATIONS

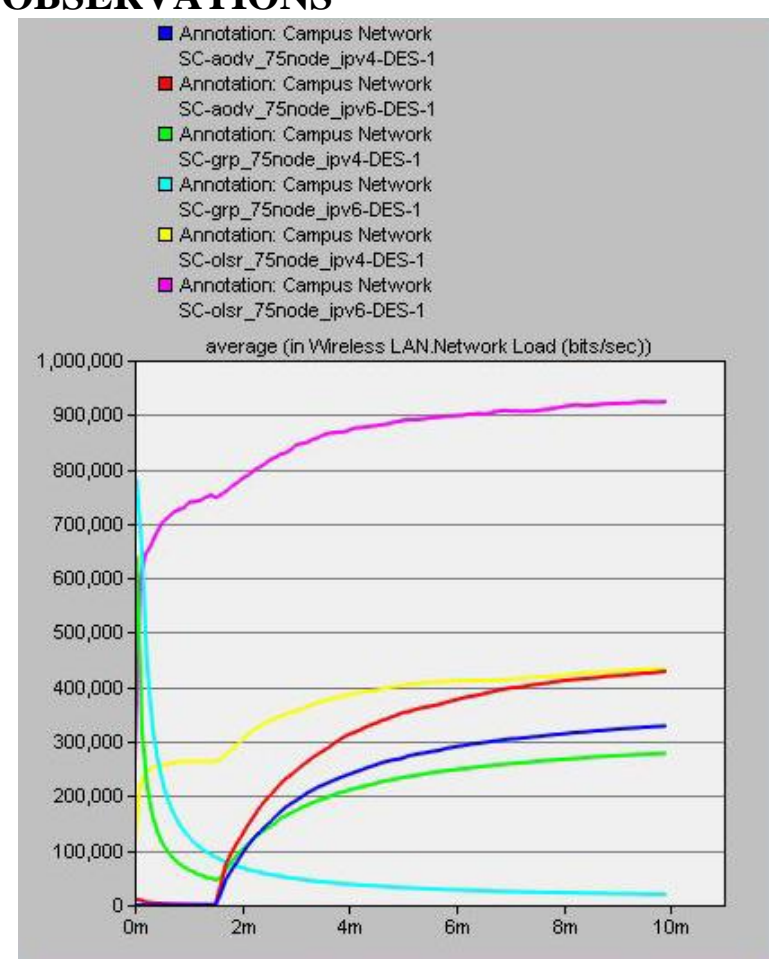

Figure 4: WLAN Network Load for 75 Nodes 


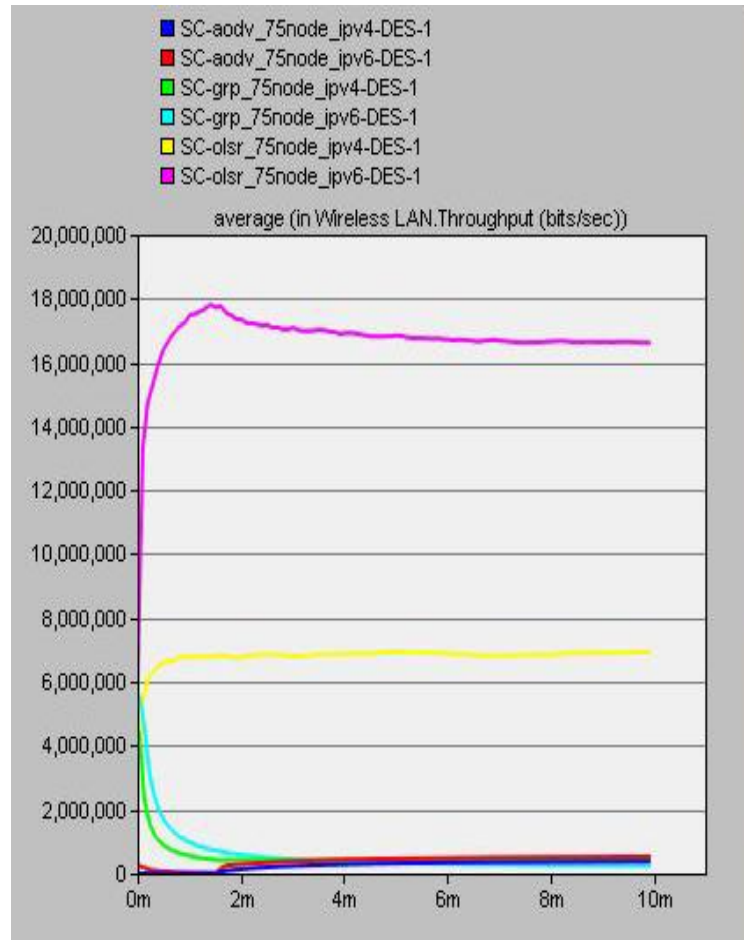

Figure 5.WLAN Throughput for 75 Nodes

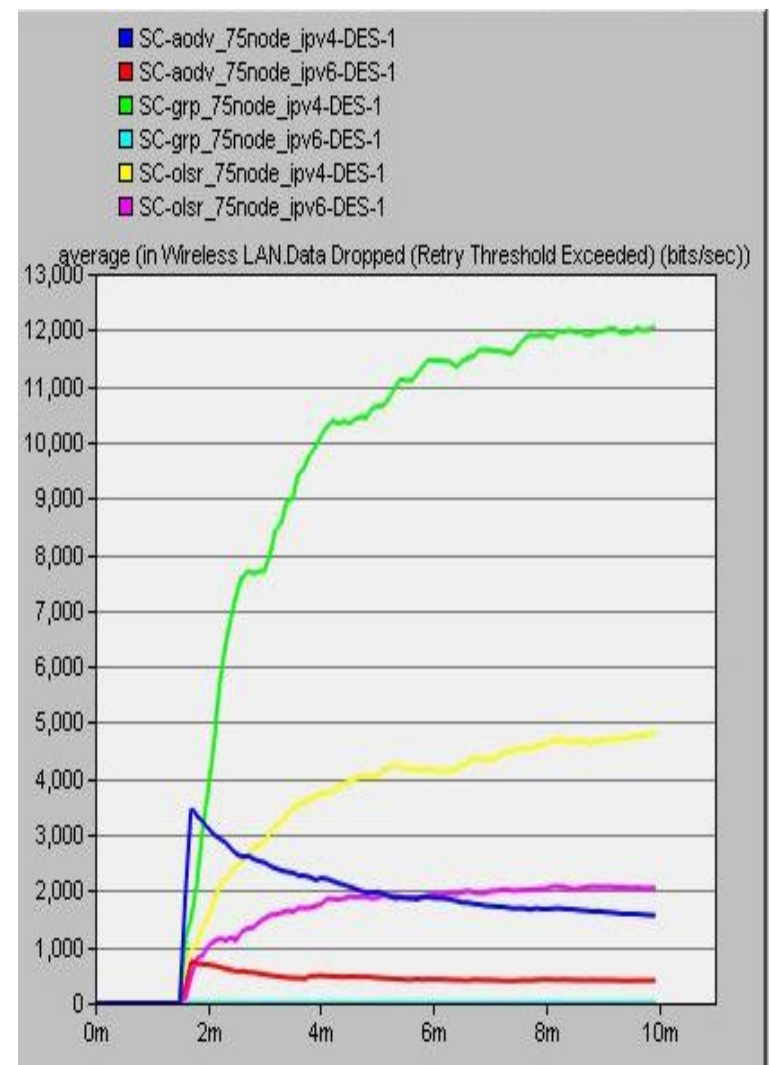

Figure 6: WLAN Data dropped for 75 Nodes

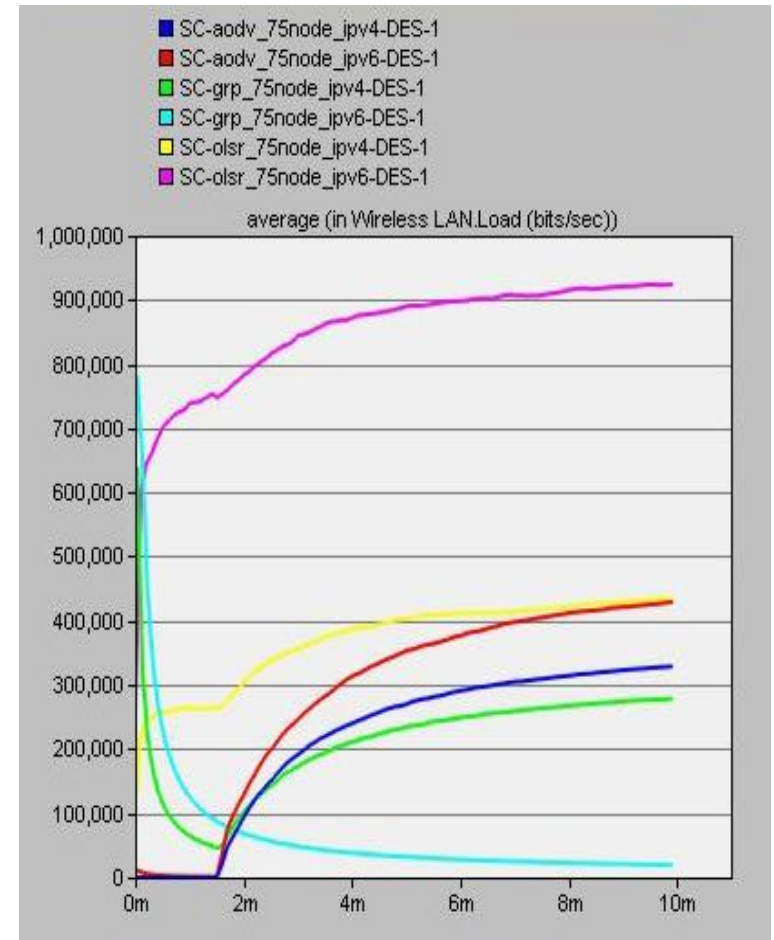

Figure 7.WLAN LAN load attempts for 75 Nodes

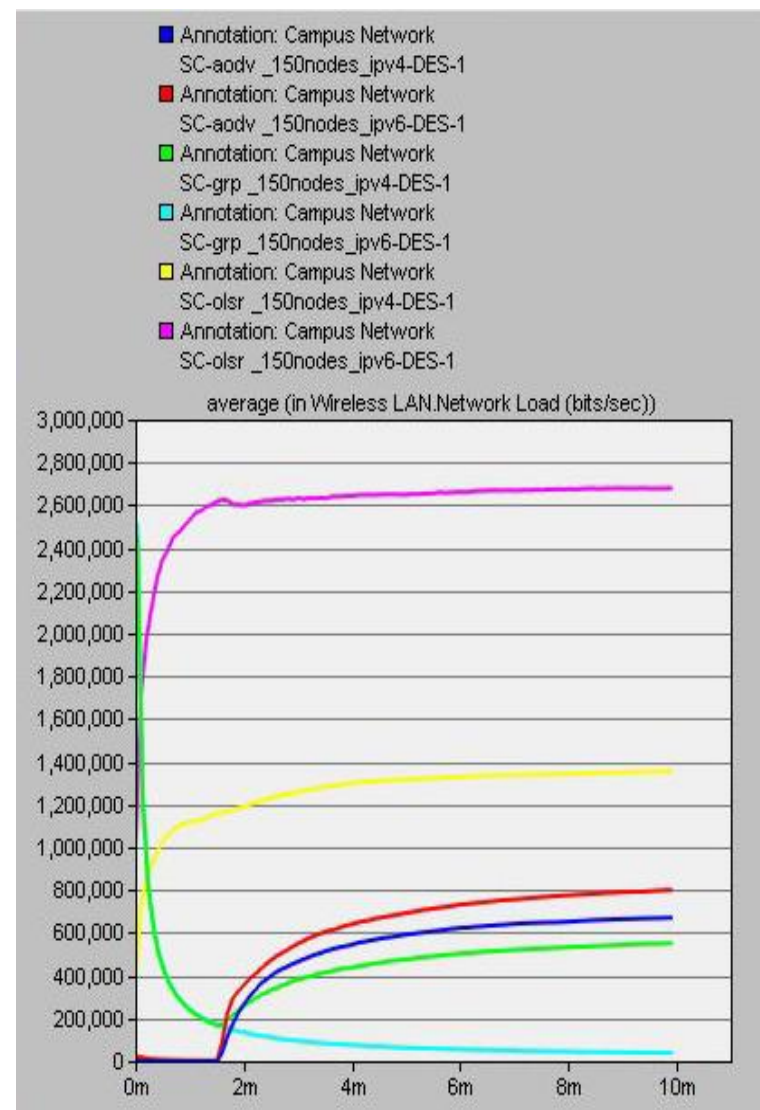

Figure 8.WLAN Network Load for 150 Nodes 


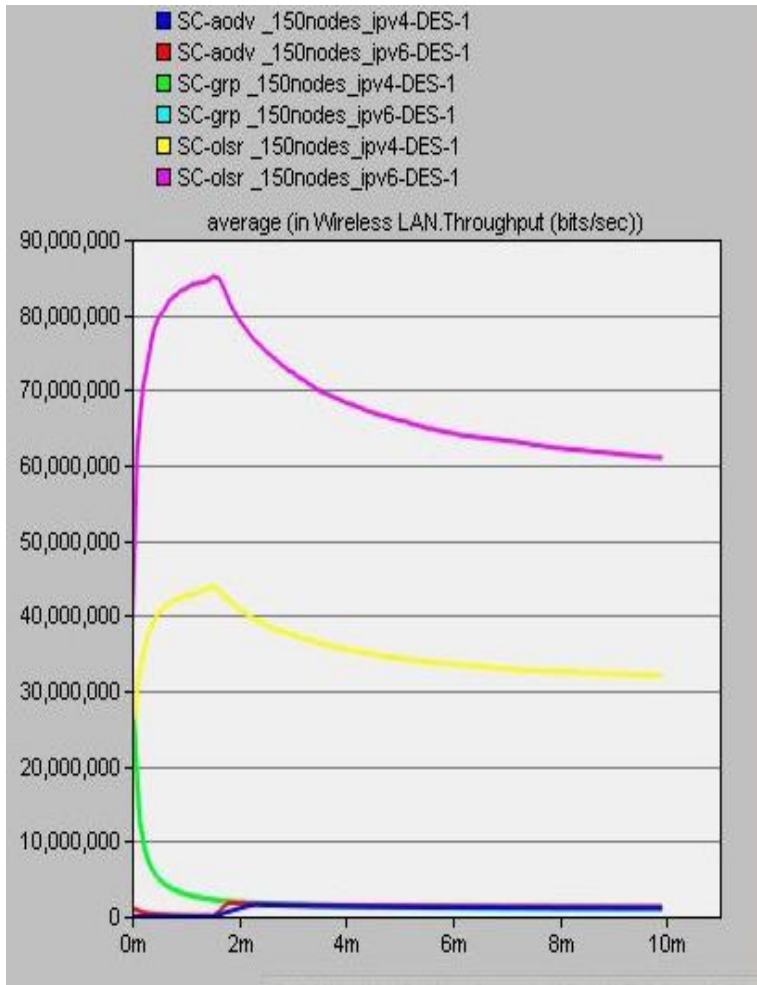

Figure 9.WLAN Throughput for 150 Nodes

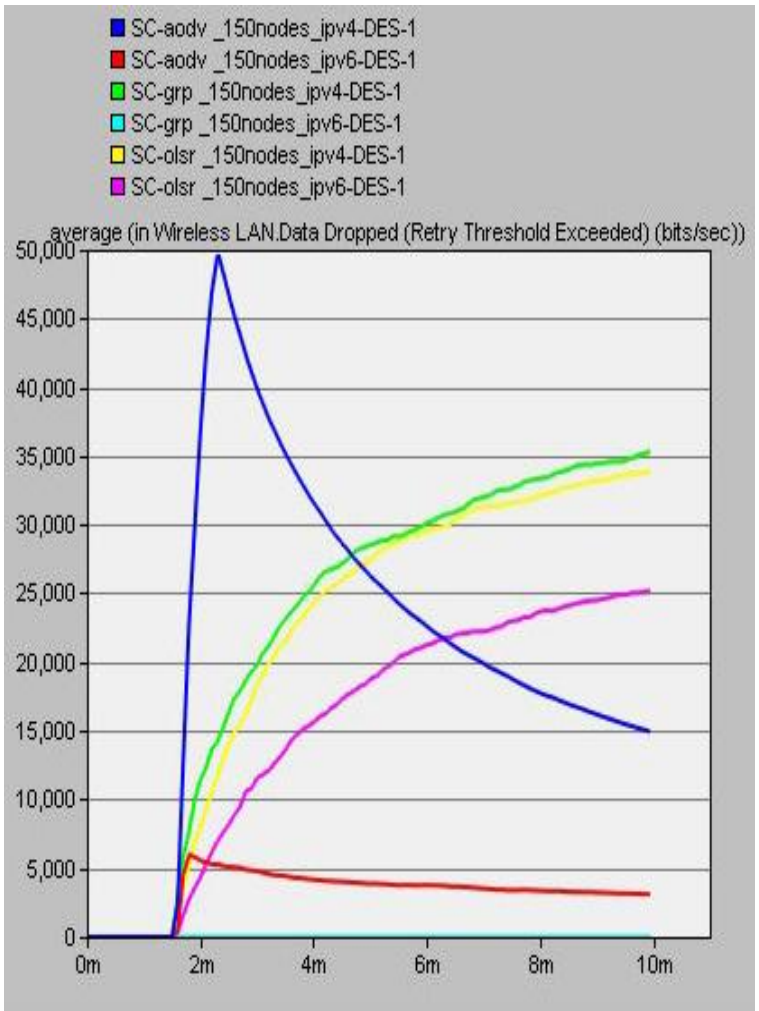

Figure 10: WLAN Data Dropped for 150 Nodes

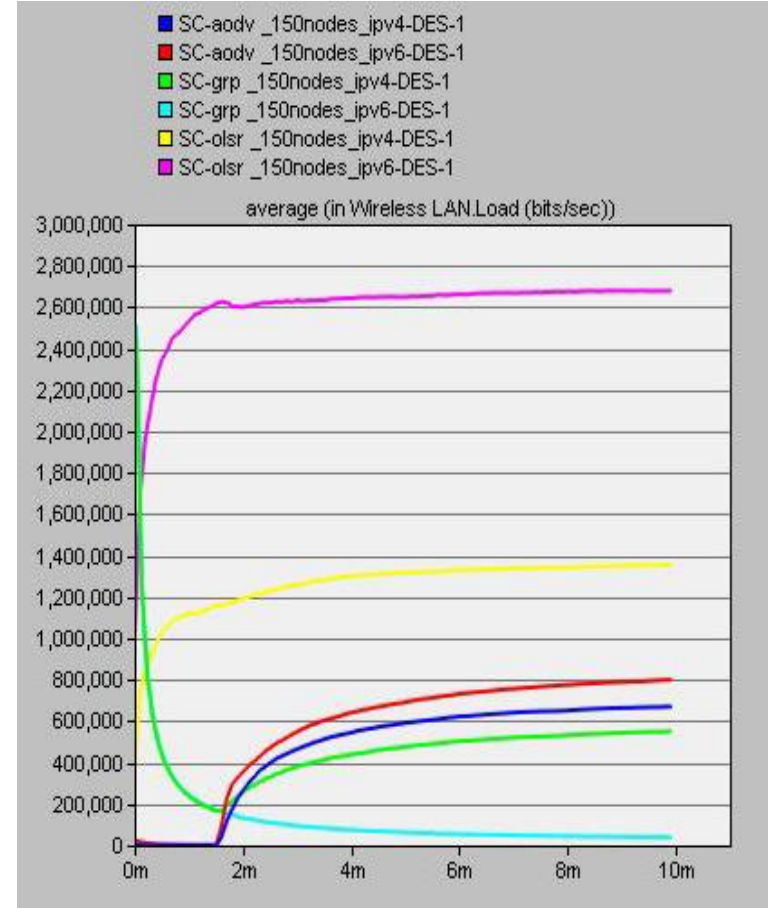

Figure 11: WLAN Lan Load for 150 Nodes

\section{CONCLUSION}

In this research we tested three routing protocols of mobile ad hoc networks OLSR, AODV, GRP under IPv4 and IPv6 environment. On the basis of observation, we say that OLSR performs better in terms of throughput and network load. Thus we conclude that OLSR performs better as compared to AODV and GRP under IPv6 environment. The results shows that GRP not perform well IPv6 environment and not suitable for the IPv6 environment. Results also show that AODV performance well in data dropped Under IPv6 environment. However, it is not necessary that OLSR always perform better the results may vary by varying networks. Every protocol being simulated using the same parameters that had been discussed to ensure the simulation produced accurate results. Table 6 summarized the performances comparison of the three routing protocols for mobile ad hoc networks under IPv6 environment. " $A$ " denotes for the best performance while " $C$ " for the worst performance.

Table 6: Performances Comparison of Three Kinds of Routing Protocols for Mobile Ad Hoc Networks under IPv6.

\begin{tabular}{|c|c|c|c|}
\hline Metrics & AODV IPV6 & OSLR IPV6 & GRP IPV6 \\
\hline Throughput & B & A & C \\
\hline N/w Load & B & A & C \\
\hline Lan Load & B & A & C \\
\hline $\begin{array}{c}\text { Data } \\
\text { Dropped }\end{array}$ & A & B & C \\
\hline
\end{tabular}

OLSR perform better than the both routing protocols under IPv6 environment and GRP perform worst in comparison with both protocols. The results may deviate in different networks. 


\section{REFERENCES}

[1] S. Deering and R. Hinden, "Internet protocol, version 6 (IPv6) specification,” IETF, RFC 2460, 1998.

[2] Hinden, R. Hindon, and S. Deering, "Internet protocol version 6 addressing architecture," IETF, RFC 3513, 2003.

[3] T. Narten, E. Nordmark, and W. Simpson, "Neighbor discovery for IP version 6 (IPv6)," IETF, RFC2461, 1998.

[4] S. Thomson and T. Narten, "IPv6 stateless address autoconfiguration," IETF, RFC 2462, 1998.

[5] C. Perkins, J. Malinen, R. Wakikawa, E. Belding-Royer, and Y. Sun, "IP address autoconfiguration for ad hoc networks," IETF Internet Draft, draft-ietf-manetautoconf-01.txt, 2001.

[6] R. Wakikawa, J. Malinen, C. Perkins, A. Nilsson, and A. Tuominen, "Global connectivity for IPv6 mobile ad hoc networks," IETF Internet Draft, draft-wakikawa-manetglobalv6-03.txt, 2003

[7] I. K. Park, Y. H. Kim, and S. S. Lee, "IPv6 address allocation in hybrid mobile ad hoc networks," in Proc. 2nd IEEE Workshop Softw. Technol. Embedded Ubiquitous Comput. Syst., May 2004, pp. 58-62.

[8] A.B. Malany, V.R.S. Dhulipala, R.M.Chandrasekaran , "Throughput and Delay Comparison of MANET Routing Protocols", Int. J. Open Problems Compt. Math., ICSRS Publications , ISSN 1998-6262,Vol. 2, No. 3, September 2009, pp. 461-468.

[9] M. Ramakrishnan, M.A. Baghyavenil, S. Shanmugavell, "Dynamic Reconfigurable Routing for High throughput in MANET", IEEE - ICSCN 2007, 22-24 February 2007, MIT Campus, Anna University, Chennai, India, pp.541544.

[10] Josh Broach, David A.Maltz, David B.Johson, Yih-Chun $\mathrm{Hu}$ and Jorjeta Jetcheva (1998). A Performance Comparison of Multi-hop Wireless Ad Hoc Network Routing Protocols. Proc. of MobiCom. Texas. USA, 1998 .

[11]Sampo Naski" Performance of Ad Hoc Routing Protocols: Characteristics and Comparison", Seminar on Internetworking. Helsinki University of Technology,2004.

[12] Mamoun Hussein Mamon (2007). "Important Characteristic of Differences between DSR and AODV Routing Protocol". MCN Conference,2007.
[13] Bu Sung Lee, Mai Ngoc San, Teck Meng Lim, Chai Kiat Yeo \& Boon Chong Seet (2006). "Processing Delay as a New Metric for On-Demand Mobile Ad Hoc",2006.

[14] S. Deering ,R. Hinden, "Internet protocol, version 6 (IPv6) specification,” IETF, RFC 2460, 1998.

[15] Hinden, R. Hindon, , S. Deering, "Internet protocol version 6 addressing architecture," IETF, RFC 3513, 2003.

[16] Wells Paris, "Ad Hoc Wireless Network Comparision A Comparision between DSR and AODV Routing Protocols" - Wireless Data Communications System, School of Engineering and Design, Brunel University.

[17] Qasim Nadia, Said Fatin \& Aghvami Hamid - "Mobile Ad Hoc Networking Protocol's Evaluation through Simulation for Quality of Service." - IAENG International Journal of Computer Science, 36:1, IJCS_36_1_10, 17 February, 2009.

[18] T.H. Clausen et al, - The optimized link state routing protocol evaluating through experiments and simulationll,mindpass center for distributed system,Aalborg university ,Denmark.

[19] C. Ahn, et.al , - Gathering-based routing protocol in mobile ad hoc networks, Computer Communications\| 30 (1) (2006) 202-206

[20] Huhtonen A,"Comparing AODV And OLSR Routing Protocols" Helsinki University of Technology Sjökulla, 2004-04-26.

[21] Razan Al-Ani -Simulation and performance analysis evaluation variant MANET routing protocol International Journal of Advancements in Computing Technology, Volume 3, Number 1, February 2011.

[22] Kumar Tanuja," Performance Evaluation Of AODV And OLSR Under Mobility” Rutgers University, 2009.

[23] Amritbir Singh "Comparative Analysis of OLSR and AODV under IPv6 Environment" International Journal of Computer Applications (0975 - 8887) Volume 61No.4, January 2013.

[24] S. R. Das, R. Castaeda, J. Yan, "Simulation-based performance evaluation of routing protocols for mobile ad hoc networks", Mobile Networks and Applications, Vol. 5, pp.179-189, 2000.

[25] Aboelela Emad, Ph. D. "Chapter 15 - Mobile Wireless Networks:Network Simulation Experiments Manual" 2nd Edition, Morgan Kufman Publishers, 30 Corporate Drive, Suite 400, Burlington, MA 01803, USA. 2008 Elsevier Inc., ISBN: 978-0-12-373974-2 